Relations industrielles

Industrial Relations

\title{
Changement planifié et développement des organisations, sous la direction de Roger Tessier et Yvan Tellier, Paris, et Montréal, Édition conjointe EPI-IFG, 1973, 825 p.
}

\section{Laurent Bélanger}

\section{Volume 29, numéro 4, 1974}

URI : https://id.erudit.org/iderudit/028574ar

DOI : https://doi.org/10.7202/028574ar

Aller au sommaire du numéro

Éditeur(s)

Département des relations industrielles de l'Université Laval

ISSN

0034-379X (imprimé)

1703-8138 (numérique)

Découvrir la revue

Citer ce compte rendu

Bélanger, L. (1974). Compte rendu de [Changement planifié et développement des organisations, sous la direction de Roger Tessier et Yvan Tellier, Paris, et Montréal, Édition conjointe EPI-IFG, 1973, 825 p.] Relations industrielles /

Industrial Relations, 29(4), 893-895. https://doi.org/10.7202/028574ar

Tous droits réservés @ C Département des relations industrielles de l'Université Laval, 1974
Ce document est protégé par la loi sur le droit d'auteur. L’utilisation des services d’Érudit (y compris la reproduction) est assujettie à sa politique d'utilisation que vous pouvez consulter en ligne.

https://apropos.erudit.org/fr/usagers/politique-dutilisation/ 
différents auteurs de même que les différences et similitudes. Le lecteur doit donc faire un effort additionnel pour mieux saisir la portée de tous ces travaux effectués à partir de cadres de référence particuliers à chacun des auteurs.

Sans doute, l'introduction rédigée par les éditeurs peut aider mais ce n'est pas suffisant. Ces derniers n'ont pas jugé bon de procéder à une réflexion d'ensemble qui aurait pu servir de conclusion à leur ouvrage.

\section{Laurent BELANGER}

Université Laval

Changement planifié et développement des organisations, sous la direction de Roger Tessier et Yvan Tellier, Paris, et Montréal, Edition conjointe EPIIFG, 1973, 825 p.

C'est beaucoup plus une présentation générale de cet ouvrage que nous voulons faire ici qu'une revue critique de chacun des articles écrits par différents auteurs dont la plupart sont québécois. L'ouvrage est un recueil de trente-sept articles auxquels on ajoute le compterendu d'une table ronde. Ces articles sont regroupés sous quatre grandes dimensions qui constituent les parties du recueil : la théorie du changement planifié et du développement des organisations; la pratique du changement planifié; le rôle de la recherche appliquée dans ce domaine; l'évaluation critique des fondements conceptuels qui soustendent la théorie et la pratique de l'intervention psychosociologique.

Dans l'introduction à leur recueil, les auteurs prennent soin de distinguer entre «changement planifié » au sens large et «changement planifié 》 au sens restreint du terme. La première signification regroupe tous les efforts systématiques de transformation sociale à l'échelle d'une société ou d'une communauté; la seconde origine du courant américain de la recherche-action et de la dynamique de groupe. Elle se confond actuellement avec le courant récent qu'on a appelé le D.O. ou «développement des organisations ». $\mathrm{Si}$ ce courant porte le nom de «planned changed $>$ ou changement planifié, il faut souligner que le changement ne se conçoit pas et ne s'implante pas de fa- çon autoritaire ou unilatérale comme dans le cas des changements pensés et conçus par des comités de planificateurs qu'on retrouve dans différents organismes de grande envergure ou de comités composés de technocrates au service des gouvernements. Au contraire, le développement des organisations (changement planifié, intervention psychosociologique) s'effectue sous un mode démocratique ou participatif, en impliquant les gens concernés dans le diagnostic de leurs problèmes et l'élaboration des changements à apporter.

Comme il n'existe pas de théorie générale du changement planifié, les auteurs parlent plutôt d'orientations théoriques dans la présentation de la première partie de l'ouvrage.

Sous ces orientations théoriques, on retrouve d'abord un essai de classification des expériences de changement planifié. Un second article traite du «sens de la complexité dans les organisations ». Dans un troisième, l'auteur, Roger Tessier, dresse «un tableau d'une stratégie de changement susceptible d'accroître le rendement de l'organisation bureaucratique dans une société en changement $\gg$ (p. 12). L'article de Yvan Tellier reprend le traditionnel débat sur le conflit 《line-staff » en l'analysant sous l'angle des rapports entre le pouvoir et le savoir. Richard Bekkard, un auteur américain, reprend le thème du malaise des cadres au sein des grandes organisations et du changement dans les systèmes de valeurs propres à cette catégorie. François Allaire traite du groupe de travail en effectuant une analogie entre ce dernier et les organisations. Dans «Experiencing et changement social», Jean Garneau essaie de démontrer qu'il est possible «de transposer au niveau $\mathrm{du}$ fonctionnement des groupes, les constatations faites au plan individuel sur les conditions favorisant le mouvement au sein du processus d'expériencing individuel $\gg$ (p. 13).

D'autres articles, dans cette même partie, reprennent le thème des stratégies et des tactiques de changement; des conditions d'implantation des changements; enfin, des modes d'influence qui peuvent s'établir entre les agents de changement et les systèmes-clients.

Les articles qui nous apparaissent les plus pertinents à une description des 
orientations théoriques sont ceux qui traitent des stratégies et des conditions d'implantation du changement. Les autres sur le malaise des cadres et la complexité des organisations relèvent beaucoup plus de l'analyse psychologique et sociologique des organisations. Tout au plus, elles peuvent servir de préalable à la compréhension du développement des organisations.

Dans la seconde partie qui traite de la pratique du changement planifié, on retrouve la description d'expériences faites à l'Alcan, au Centre pilote Laval. On y décrit aussi l'apprentissage de certaines techniques utilisées dans un effort de changement planifié, telles que la formation en laboratoire, l'analyse des rôles, le processus rationnel de solution des problèmes. Deux articles traitent plus spécifiquement de la nature de la consultation dans une expérience de développement organisationnel.

Dans la troisième partie, le rôle de la recherche appliquée dans une expérience de changement planifié est étudié sous deux aspects: celui d'un outil intervention au cours du diagnostic de la situation à changer et au cours du suivi, c'est-à-dire, au moment d'évaluer les résultats de l'expérience pour apporter des corrections ultérieures. Les deux premiers articles sont de Roger Tessier. Il tente d'abord, d'établir les grandes lignes d'une recherche visant à décrire et expliquer les effets de l'intervention psychosociologique de quelque nature qu'elle soit. Ensuite, il présente les résultats d'une recherche faite pour le compte du ministère de l'Education dans le cadre de l'expérience SEMEA (Stage d'Entraînement aux Méthodes d'Education Actives).

Dans un troisième article, Jean-Pierre Lanthier et Yvon Rodrigue donnent un compte-rendu des principaux travaux de recherche visant à identifier et expliquer l'impact des expériences de formation en relations humaines; en particulier, les sessions de dynamique de groupe ou groupe de base. Ils s'intéressent, en plus, aux recherches faites sur les aspects méthodologiques de ce type de formation, sur les ressources utilisées au point de départ dans les programmes, de même que sur le déroulement de ces expériences. Jocelyne Savoie, dans un autre article, s'interroge sur l'utilité des efforts d'évaluation dans le domaine de la formation aux relations humaines. Elle traite aussi des méthodes de recherche propres à ce type d'évaluation. Jacques Perron, à son tour, s'interroge plutôt sur le rôle des valeurs dans la recherche. Robert Poupart décrit les interractions ou la nature idéale des échanges entre ceux qui utilisent le savoir et ceux qui le produisent, de sorte que la recherche au lieu d'être conçue uniquement en vase clos originerait aussi du monde de l'action.

Dans un dernier article, François Gagné décrit les problèmes posés par l'utilisation du test Perpe (test permettant de tracer un profil de la relation maîtreélève). Il s'interroge aussi sur l'aptitude de cet instrument à effectuer des changements au plan des attitudes et comportement pédagogique.

Comme nous l'avons mentionné plus haut, la quatrième partie, qui est la plus courte, se veut une réflexion sur les valeurs sous-jacentes aux expériences de changement planifié. Les auteurs reprennent certaines critiques faites à l'endroit de la dynamique des groupes, en particulier celles qui considèrent ce mouvement comme un instrument subtil d'intégration de l'individu au groupe ou aux organisations; ou encore, cornme un instrument de préservation du statu quo.

La meilleure façon d'aborder la lecture de cet ouvrage, c'est, à mon avis, d'oublier les rubriques qui servent à regrouper les articles et d'aller directement à ceux dont les sujets nous préoccupent. En effet, les articles proviennent d'expériences et de réflexions tellement différentes qu'il est impossible de se faire une vue d'ensemble de l'une ou l'autre des dimensions retenues. Cette manière d'aborder la lecture de l'ouvrage nous permet de mieux saisir la contribution de chaque auteur, et chacune de ces contributions m'apparaît valable qu'elle soit du domaine du développement des organisations, de l'analyse organisationnelle, de la dynamique du groupe, ou encore de la recherche en sciences du comportement.

Une dernière remarque générale porte sur le choix des articles. Un nombre assez important réfère au milieu de l'éducation, puisque c'est surtout dans ce secteur que les auteurs ont acquis une certaine expérience pratique. Exception faite de l'expérience d'Alexander Winn 
à l'Alcan, aucun article ne relate une expérience vécue de D.O. dans le milieu de l'entreprise privée. C'est assez paradoxal lorsqu'on songe à la multiplicité et à la diversité des expériences de D.O. faites tant aux Etats-Unis qu'au Canada dont, au moins, quelques-unes dans la province de Québec.

\section{Laurent BELANGER}

\section{Université Laval}

Career Management, by Marion S. Kellog, New York, American Management Association, 1972, 200 p.

Le développement de la carrière des employés salariés (cadres, professionnels, ou équivalents) se présente sous un nouvel aspect depuis quelques années. Les règles du jeu ont été sensiblement modifiées. De moins en moins de corporations ont maintenant la réputation d'offrir une sécurité d'emploi à vie pour leurs employés; de plus en plus de jeunes diplômés universitaires accordent plus de loyauté à leur profession plutôt qu'à leur employeur initial.

Ces nouvelles règles de jeu sont à la base de l'ouvrage de Kellog. A partir de ce point de référence, l'auteur dégage les implications qui en résultent tant pour l'employé que pour l'employeur.

Aujourd'hui l'employé est le premier responsable de sa carrière. Il ne peut plus ou ne doit plus s'en remettre à la discrétion de son supérieur en espérant que les événements et un effort soutenu le favorisent. Il doit d'abord déterminer sa propre orientation, ses objectifs de carrière puis en faire part à l'organisation dont il fait partie et vérifier périodiquement si elle peut toujours satisfaire ses attentes.

De son côté, l'organisation doit soutenir et faciliter ces efforts de la part de ses membres. Distinguant fort judicieusement la planification de maind'œuvre de la planification de carrière, Kellog présente diverses politiques et pratiques administratives qui doivent conduire à l'intégration des deux dans un effort total de planification et de développement des ressources humaines.

Sans être nécessairement d'accord avec chacune de ces politiques et pratiques, dans l'ensemble nous y retrou- vons plusieurs éléments d'intérêt que nous apprécions.

Comme la majorité des ouvrages publiés par l'AMA, ce volume s'adresse davantage aux praticiens qu'aux théoriciens, les premiers devant se recruter cette fois non seulement chez les directeurs du personnel mais aussi parmi les administrateurs, ceux qui ont la responsabilité immédiate de personnel. Egalement les employés qui sont les premiers concernés par le sujet devraient s'intéresser à ce volume. Kellog présente plusieurs idées qui requièrent une nouvelle conception du développement de carrière tant pour les salariés que pour leurs supérieurs. C'est en quelque sorte le défi qui s'offre à tous ceux qui veulent d'une part un travail intéressant et motivant, un sentiment de réalisation de soi-même et de croissance, d'autre part un personnel qualifié, intéressé, et prêt à faire face à de nouvelles responsabilités pour éviter la routine et la facilité.

\section{Alcan, Arvida}

\section{Jean-Pierre BEAULIEU}

Equity and Efficiency Effects from Manpower Programs, by Corry F. Azzi, Toronto, D.C. Health and Co., 1973, $97 \mathrm{pp}$.

Fondamentalement, cet ouvrage traite d'un des types possibles de l'évaluation des programmes de main-d'œuvre, l'analyse coûts-bénéfices. Cependant, l'approche utilisée se veut différente comme le laisse voir cette citation du premier paragraphe du livre:

«Although the recent development and proliferation of federally subsidized manpower programs designed to benefit the disadvantaged has received considerable attention, studies which have attempted to quantify the costs and benefits of these programs have been inadequate $\gg$.

$\mathrm{La}$ base de ce reproche réside dans le fait que ces études ont traditionnellement supposé que les salaires égalaient la valeur du produit marginal, ce qui, selon Azzi, est un modèle très irréaliste du comportement de la firme.

Contrairement à ce que peut laisser croire son titre, ce livre ne traite pas des programmes de main-d'œuvre, mais plutôt exclusivement de programmes de formation professionnelle en cours 